\title{
Tutoría universitaria y educación intercultural: debates y experiencias
}

\section{University tutoring and intercultural education: debates and experiences}

\begin{abstract}
Nuria Macarena Rodríguez es Becaria Doctoral del Consejo Nacional de Investigaciones Científicas y Técnicas (CONICET) en el Instituto de Investigaciones en Ciencias Sociales y Humanidades (ICSOH). Universidad Nacional de Salta (Argentina) (nuria.macarena.rodriguez@gmail.com) (D) (https://orcid.org/0000-0003-3692-294X)
\end{abstract}

Dra. María Macarena Ossola es Investigadora Asistente del Consejo Nacional de Investigaciones Científicas y Técnicas (CONICET) en el Instituto de Investigaciones en Ciencias Sociales y Humanidades (ICSOH). Universidad Nacional de Salta (Argentina) (macossola@gmail.com)(iD(https://orcid.org/0000-000 1-7222-7464)

Recibido: 2019-01-30 / Revisado: 2019-05-07 / Aceptado: 2019-05-15 / Publicado: 2019-07-01

\section{Resumen}

Este artículo reflexiona sobre los significados que asume la tutoría universitaria para los estudiantes indígenas y tutores pares que han participado de esta instancia durante el desarrollo de sus carreras de grado en la Universidad Nacional de Salta (Argentina). El objetivo es reconocer cuáles son los aportes de la tutoría en la retención y graduación universitaria de las y los jóvenes indígenas, como así también su impacto en la formulación de una propuesta educativa intercultural a nivel universitario. La metodología es cualitativa y se analiza un estudio de caso en el que se reconstruyen parcialmente las trayectorias socio-escolares de dos estudiantes indígenas y dos tutores pares. Los resultados señalan que las tutorías conforman un espacio formativo en sí mismo, donde se transmiten conocimientos y habilidades de las cuales los estudiantes se apropian en diferentes momentos de sus trayectorias académicas y laborales. En las conclusiones se destaca que las tutorías para pueblos originarios constituyen un ámbito en el cual las relaciones interculturales se construyen y negocian cotidianamente.

Descriptores: Educación intercultural, universidad, tutoría, estudiantes, población indígena, juventud.

\begin{abstract}
This article reflects about the meanings which assumes the University Tutoring for indigenous students and tutors peer, who have participated of this instance during their degree courses development at the National University of Salta (Argentine). The objective is to recognize what are the contributions of the tutoring in the retention and university graduation of the indigenous youth, as well as its impact on the formulation of an intercultural education proposal at the university level. The methodology is qualitative, and a case study is analyzed in which the socio-school trajectories of two indigenous students and two tutors peer are partially reconstructed. The results indicate that the tutoring make up a formative space in itself, where knowledge and skills are transmitted from which the students appropriate at different times of their academic and work trajectories. In the conclusions it is emphasized that the tutoring for indigenous peoples constitute an area in which intercultural relations are built and negotiated on a daily basis.
\end{abstract}

Keywords: Intercultural education, university, tutoring, students, indigenous peoples, youth.

Forma sugerida de citar: Rodríguez, N., \& Ossola, M. M. (2019). Tutoría universitaria y educación intercultural: debates y experiencias. Alteridad, 14(2), 172-183. https://doi.org/10.17163/alt.v14n2.2019.02 


\section{Introducción}

Argentina históricamente se ha identificado como un país blanco, descendiente de europeos, negando e invisibilizando a los pueblos originarios que habitan en su territorio (Briones, 2005). Sin embargo, la reforma constitucional de 1994 reafirmó sus derechos, reconociendo su preexistencia étnica y cultural, y garantizando el respeto a su identidad, a una educación intercultural bilingüe, al reconocimiento de las personerías jurídicas de sus comunidades y a la posesión comunitaria de las tierras que ocupan. El Informe Anual de Pueblos Indígenas 2014 señala que, durante los últimos años, es notable en Argentina el aumento de la conciencia indígena acerca de la pertenencia a un pueblo. Esto tiene como efecto el incremento de pueblos indígenas reconocidos (Mikkelsen, 2014). Así, mientras en 2001 había 600329 personas que se reconocían pertenecientes o descendientes en primera generación de un pueblo indígena (INDEC, 2001), en 2010 existían 955032 personas en la misma condición (INDEC, 2010).

La temática de la Educación Superior de los Pueblos Indígenas comenzó a tratarse en Argentina a partir del 2000, tardíamente en comparación con otros países de la región. Esto se debe, principalmente, a que las universidades públicas se visualizan como ámbitos abiertos y accesibles, dado que su gratuidad (Rezaval, 2008; Paladino, 2009) y el tipo de políticas públicas que se implementan en este nivel, tienden a favorecer la inclusión de los grupos económicamente marginados, con una indagación tardía por las identidades de los sujetos (Claro, \& Seoane, 2005).

Sin embargo, es importante tener en cuenta que las universidades públicas argentinas desde sus orígenes - no fueron pensadas para la interculturalidad, ni para la diversidad cultural y lingüística. Son organizaciones intrínsecamente conservadoras que responden a premisas del mundo de los siglos XIX y primera mitad del $\mathrm{XX}$, que se organizan como sistemas con unidades académicas — facultades, departamentos, escuelas, institutos, etc. - con cierto grado de autonomía e identidad propia (Coronado, \& Gómez Boulin, 2015). Las universidades son instituciones que se encuentran en permanente construcción, tensadas por su diversidad y apego a la tradición. Sus objetivos han ido cambiando con el tiempo, pero mantienen un alto grado de credibilidad en la población en general, y entre los jóvenes en particular. Entrañan una promesa - no siempre clara o explícita- de un futuro valioso y más justo, que no puede ser reducido a un espacio para formarse para un empleo (Coronado, \& Gómez Boulin, 2015).

En Argentina las universidades cuentan con un ingreso irrestricto. Los estudiantes de este nivel son numerosos y, a la vez, cada vez más diversos en cuanto a intereses, procedencia y trayectorias previas. Correlativamente, la deserción constituye una preocupación central, pues son más los estudiantes que se van que los que permanecen y se gradúan. Al respecto, resulta central destacar que estas instituciones suponen una igualdad de oportunidades, lo cual no favorece a quienes pelean su ingreso y permanencia desde posiciones iniciales de desventaja (Coronado, \& Gómez Boulin, 2015).

Cabe aclarar que la Ley de Educación Nacional $N^{\circ} 26.206$ del 2006, establece la Educación Intercultural Bilingüe como una modalidad del sistema educativo en sus niveles inicial, primario y secundario; pero no incluye a la educación superior, que se rige por la Ley de Educación Superior No 24.521 de 1995 y por normas reglamentarias que se fueron dictando para posibilitar su aplicación (Sánchez Martínez, 2003). Esta ley no contempla la diversidad cultural, la interculturalidad ni el plurilingüismo, a pesar de haber sido sancionada en un contexto de multiculturalismo neoliberal. No obstante, en los últimos años, y merced a la autonomía que caracteriza a las universidades públicas, se han implementado diferentes iniciativas de base étnica (Ossola, 2016), siendo diecinueve las universidades nacionales que cuentan con alguna política institucional para los estudiantes de pueblos indígenas (Guaymás, 2016). 
En Salta, provincia ubicada al norte de Argentina, limitando con seis provincias (Jujuy, Catamarca, Tucumán, Santiago del Estero, Chaco y Formosa) y tres países (Paraguay, Bolivia y Chile), hay 79204 personas que se autorreconocen como indígenas o descendientes de algún pueblo originario, sobre un total de 1 214441 habitantes. Esto representa el 6,5\% de la población, muy por encima del $2,4 \%$ a nivel nacional (INDEC, 2010). Además, esta es la provincia que presenta la mayor diversidad de pueblos originarios: Kolla, Wichí, Guaraní, Ava Guaraní, Weenhayek, Chané, Chorote, Qom (Toba), Chulupí, Tapiete, Diaguita-calchaquí, Tastil, Iogys, Atacama y Lule. ${ }^{1}$

La Universidad Nacional de Salta (UNSa) se define en sus Bases Fundacionales (1972) como una "institución académica de frontera" (Ossola, 2015; Hanne, 2018), por la impronta de su ubicación geopolítica y la diversidad que caracteriza a la provincia. Además, es una Universidad de "tradición plebeya" (Carli, 2012), en tanto recibe estudiantes de niveles socioeconómicos medios y bajos. Es importante destacar que la mayor parte de sus estudiantes son la primera generación familiar en acceder a este nivel educativo (Ilvento, Martínez, Rodríguez, \& Fernández Berdaguer, 2011). Desde 2010 la UNSa cuenta con un "Proyecto de tutorías con los estudiantes de Pueblos Originarios", que contempla la actuación de tutores pares, en el acompañamiento a los estudiantes indígenas que lo deseen. Estas tareas incluyen un abordaje socioafectivo y académico, en pos de lograr su retención y graduación.

En este artículo se analizan las experiencias de estudiantes y egresados que han participado en este proyecto, con el objetivo de comprender la incidencia de las tutorías en su formación socio-académica.

\subsection{Inclusión e interculturalidad en la universidad}

Existen múltiples definiciones para términos como inclusión y exclusión, por lo cual resulta necesario clarificar el sentido en que son enten- didos. Si bien intentar precisar las categorías inclusión y exclusión no se limita a revisar o acordar una definición, sino que es el marco teórico junto con el contexto y los usos que se realicen de ellas lo que va a definir sus significados nunca acabados (Diez, García, Montesinos, Pallma, \& Paoletta, 2015), resulta conveniente esbozar algunas ideas para un análisis que no pretende ser finalizado, sino problematizador.

Por un lado, para hablar de inclusión es necesario hacer referencia a la exclusión, porque la primera es consecuencia del reconocimiento de la existencia de la segunda y de la importancia de intervenir para atender a quienes resultan excluidos (Wigdorovitz de Camilloni, 2008). Entonces, ¿Quiénes son los excluidos? Los pobres, los pobladores de zonas rurales remotas, las poblaciones indígenas, las minorías lingüísticas y religiosas, las personas con discapacidad, quienes padecen enfermedades contagiosas, todos aquellos que son diferenciados de los demás y - por tal razón- pesa sobre ellos un estigma social (Wigdorovitz de Camilloni, 2008).

Por otro lado, resulta importante aclarar que no existe una relación proporcional entre exclusión e inclusión, sino que son procesos interrelacionados que ocurren simultáneamente. Mayores acciones de inclusión no disminuirán proporcionalmente las formas de exclusión e incluso pueden generar otras manifestaciones de exclusión. De hecho, pensar en que un grupo plantea acciones de inclusión para otro, representa un modo de exclusión, en tanto fortalece ciertas relaciones de dominación.

Respecto a la posibilidad de escolarización, los sentidos de la inclusión no se producen en el vacío, sino que forman parte de las diferentes visiones respecto de los motivos y los logros que se persiguen a través de la educación formal. Esto es más significativo si se piensa en la educación superior, que no es obligatoria. Además, implica reconocer que la inclusión y la exclusión son categorías analíticas que, en ciertas ocasiones, no llegan a dar cuenta de la complejidad de las trayectorias socio-escolares reales de los sujetos, 
quienes se apropian de los espacios académicos de formas particulares, $\mathrm{y}$ en base a condiciones de existencia inequitativas y desiguales.

Otros conceptos a precisar refieren a las situaciones llamadas de interculturalidad y/o diversidad cultural, construcciones históricas, sociales y académicas que se encuentran en permanente disputa y negociación (Briones, Delrio, Lanusse, Lazzari, Lorenzetti, Szulk, \& Vilvaldi, 2006) y por ello no deben ser tomadas como datos de la realidad, sino como palabras clave o palabras-llave, a través de las cuales se hace posible reflexionar acerca de cómo son imaginados los vínculos entre los diferentes sectores que conforman la sociedad. Se considera que los estudios sociales que indagan situaciones de interculturalidad deben incluir las arenas políticas en las que se llevan adelante las disputas por sus sentidos, asumiendo lo intercultural como un signo ideológico, para luego rastrear los regímenes de verdad que alientan las nociones de sentido común tras las que se esconden relaciones de asimetría social y simbólica (Briones et al., 2006).

Lo anterior es importante si se toma en consideración que, en los últimos años, en varios países, se pasó del discurso de homogeneización cultural a un reconocimiento de la diversidad étnica, otorgando una serie de derechos específicos a ciertos grupos. En este punto cabe señalar que, al menos en Argentina, las políticas estatales no persiguen fines interculturales más que en su denominación. Esto es así en tanto no propician diálogos, son focalizadas en determinados grupos y formuladas muchas veces sin la participación de los sectores destinatarios. Es decir que se reconocen las identidades étnicas, pero no se generan condiciones para la modificación de las relaciones sociales (Diez, 2004).

Por lo expuesto, se torna necesario reflexionar acerca de la distancia que existe entre los postulados teóricos basados en la interculturalidad y las políticas públicas para la inclusión educativa (Möller, 2012) puesto que se evidencia una brecha entre los objetivos ligados a un horizonte intercultural y los efectos de las estrategias implementadas por los proyectos destinados a la inclusión (Ossola, 2015). De esta manera, programas elaborados con intención de incluir, empoderar y brindar espacios de autonomía terminan, en muchos casos, por ubicar a las poblaciones destinatarias en posiciones de subyugación que reproducen las relaciones sociales mayores (Delgado, 2002).

En el caso específico de la inclusión de estudiantes indígenas a universidades convencionales, las políticas de inclusión han sido criticadas por parte de sus beneficiarios por reproducir, al interior de la Universidad, situaciones históricas de opresión de los pueblos indígenas, tales como el tutelaje (Luciano, 2009), el clientelismo y el patronazgo (Paladino, 2006). De este modo se señala que no basta con formar indígenas para asegurar su protagonismo, si no se rompe con las diferentes formas de colonización (Luciano, 2009). De allí se desprende la importancia de aceptar que las iniciativas que buscan la inclusión en base a premisas de interculturalidad en el ámbito universitario deben considerar la desigualdad estructural de las sociedades en las que habitamos (Reygadas, 2007). Esto es importante para evitar que las diferencias estructurales se transformen en estigmas naturales (Delgado, 2002).

\subsection{Tutorías universitarias}

Existen variadas acepciones y diferentes modalidades de tutorías. La que se analiza en este trabajo corresponde a una tutoría universitaria, definida como un acompañamiento y apoyo a los estudiantes en su proceso de adquisición de aprendizajes y competencias a nivel personal y profesional (Lovato Fraile, \& Ilvento, 2013). Este proceso sucede en la universidad, pero las habilidades allí adquiridas también pueden observarse en las trayectorias laborales (y vitales, en términos más amplios). De este modo, podemos definir a las tutorías como un vínculo socio-pedagógico entre diferentes actores universitarios, tendiente a personalizar la experiencia académica de los estudiantes. 
Cuando se habla de acompañamiento, se refiere a un espacio de encuentro, de ensayo permanente, de puesta en práctica, para pensar con $y$ a otros, que media entre las condiciones reales de cada organización y las condiciones requeridas por los estudiantes (Coronado, \& Gómez Boulin, 2015). Esto permite evidenciar que no se concibe a las tutorías universitarias como cerradas ni acabadas, sino que se encuentran en constante construcción, como espacios interactivos y de vínculos interpersonales.

El momento en que se originan las tutorías institucionalizadas difiere según el nivel educativo al que se haga referencia. En el contexto universitario argentino, las tutorías existen desde los inicios mismos de las universidades, pero su función y objetivos fueron variando según el contexto histórico y sociocultural en el que se desarrollaran, así como fueron cambiando los modelos de universidad en el país. En la actualidad, la función del tutor en las universidades está vinculada a ayudar y cumplir tareas que complementan la enseñanza de los docentes; en estas modalidades hay mayor flexibilidad en el ejercicio de los roles de tutor y tutelado, donde las posiciones no son inmutables (Capelari, 2016) y las relaciones jerárquicas se difuminan.

Es importante considerar dos aspectos centrales de las tutorías que se analizan en este trabajo: 1. que se trata de tutorías entre pares, es decir que tanto los tutores como los tutelados son estudiantes de la misma universidad, posibilitando la rotación en los roles de tutores y tutelados, así como cierta simetría en las relaciones; 2. que la participación en las mismas es voluntaria, no obligatoria, lo que conlleva el reto de implicar a los estudiantes en tales espacios.

\section{Material y métodos}

Este artículo reúne conclusiones parciales de una investigación cualitativa enmarcada en la Antropología de la Educación y la Pedagogía Crítica, consistente en un estudio de caso, ${ }^{2}$ lo cual supone un análisis de lo particular y lo único, que permite dar cuenta de un contexto sociocultural determinado en un momento dado. Sin embargo, la situación analizada permite reflexionar sobre ciertas condiciones que repercuten en el caso seleccionado y también en otros similares (Grupo de Investigación: Hum 0109, 1997).

La selección del caso (Proyecto de tutorías con los estudiantes de pueblos originarios) se realizó en base a tres criterios: 1. Que el caso ofreciera mayores oportunidades de aprendizaje con respecto a la temática general (Stake, 1995); 2. La factibilidad de acceso a la información; 3 . Su exploración parcial previa (Ossola, 2015), lo cual permite realizar un estudio longitudinal en el que se comparten algunas variables de indagación.

Para la recolección de información se realizaron entrevistas en profundidad a: jóvenes estudiantes que participan o han participado de las tutorías; tutores y tutoras (indígenas y no indígenas) y a la coordinadora del Proyecto. Las entrevistas estuvieron orientadas a la reconstrucción de las trayectorias socio-escolares a partir de narrativas biográficas. Para realizar esta reconstrucción se pone el foco en las intersecciones entre biografía y educación, que permiten reconocer la importancia que tienen las instituciones escolares en la (re)elaboración de los proyectos de vida de los sujetos. Esta decisión asume que —en educación — la investigación autobiográfica genera conocimientos sobre los sujetos, pero también sobre sus vínculos sociales y territoriales (Delory-Momberger, 2009).

Indagar en la narrativa autobiográfica permite una reconstrucción parcial de las trayectorias escolares de los sujetos, entendidas como los recorridos de las personas en el sistema escolar, comparados con la expectativa que supone el diseño de tal sistema (Terigi, 2008). Gran parte de los estudios existentes se basan en datos estadísticos que permiten delinear trayectorias escolares teóricas o ideales, que señalan los recorridos de los sujetos cuya trayectoria se ajusta a la prevista o normativizada (Terigi, 2008). 
Por otra parte, están las trayectorias escolares reales que no se ajustan necesariamente a las expectativas del sistema educativo formal, pues los niños y jóvenes desarrollan su escolarización de maneras heterogéneas, variables y muchas veces imprevistas (Terigi, 2008). Para el caso en estudio, es importante señalar que entre los pueblos indígenas se evidencia un alejamiento pronunciado respecto de las trayectorias ajustadas a la normativa conforme los sujetos avanzan en edad y, más aún, cuando son correlacionados la edad y el año cursado o, específicamente, la edad y el año aprobado. De este modo, la repitencia, la sobre-edad y la deserción caracterizan las trayectorias escolares de los estudiantes indígenas en nuestro país (UNICEF, 2009), frente a lo cual surge nuevamente la pregunta por los métodos y las técnicas para obtener la información.

Como los estudios sobre trayectorias escolares disponibles se basan en los datos estadísticos de cada país, tienen una importante limitación, ya que estos datos no consideran, a modo de unidad de recolección o de análisis, a las personas. Los datos escolares más frecuentes - aquellos a partir de los cuales se delinean y evalúan las políticas públicas escolares- refieren a los sujetos, pero no son ellos la fuente de información (Terigi, 2008).

Por lo expuesto, para intentar comprender las experiencias escolares en el contexto de vida de los jóvenes indígenas y tutores de estudiantes indígenas, se ha recurrido a una lógica cualitativa de investigación. Las entrevistas biográficas, particularmente, permiten abordar las historias de vida de los sujetos, a partir de su propio relato, lo cual habilita la reconstrucción de aspectos formales de su escolaridad (edad de ingreso, años de permanencia, etc.), como así también de aspectos subjetivos (auto-valoración del desempeño escolar, causas del acercamiento o alejamiento de las instituciones, etc.).

Este análisis puede resultar eficaz para cuestionar ciertos prejuicios en torno al fracaso escolar de los niños, adolescentes y jóvenes indígenas. Estudios similares han mostrado que la escuela funciona para los pueblos originarios a través de fuerzas centrífugas y centrípetas, es decir, la escuela simultáneamente ejerce atracción y repelencia para estos pueblos (Hecht, 2010). También se ha señalado que allí se realizan relecturas globales de las experiencias individuales y colectivas previas (Aikman, 1999), a la vez que se organizan y proyectan alternativas de futuro (Czarny, 2008). La experiencia universitaria, en particular, reviste un espacio que permite reconstruir las huellas que la escolaridad ha dejado en estos pueblos (Luján, Soto, \& Rosso, 2018).

En cuanto a la selección de los sujetos a entrevistar, y en concordancia con la lógica cualitativa del estudio, se realizó un muestreo intencional mediante el cual se seleccionaron los casos por su capacidad para generar información relevante (Yuni, \& Urbano, 2016). En los estudios cualitativos, la idea de representatividad de la muestra está vinculada más a la significatividad que a la cantidad, en tanto cada uno de los informantes presenta particularidades a partir de las cuales se realiza el análisis, en este sentido, las muestras tienden a ser intensivas: se estudian pocos casos, pero en mayor profundidad (Yuni, \& Urbano, 2016).

En base a lo planteado, se seleccionaron cinco participantes, teniendo como criterio que presenten situaciones diferentes respecto al caso: 1. La autora del proyecto y coordinadora de los espacios de tutorías. 2. Dos tutoras, una perteneciente a una comunidad indígena, que aún no finalizó la carrera y que ha perdido contacto con las tutorías; y una no-indígena, que se graduó y aún colabora con las tutorías. 3. Dos tutelados que participan en la mayor parte de las actividades propuestas desde el espacio de tutorías. Resulta importante aclarar que para este artículo se priorizó retomar la palabra de las dos tutoras, teniendo en cuenta los ejes temáticos desarrollados.

\section{Análisis de datos}

\subsection{Tutoría como espacio formativo}

Para este caso particular de estudio, se ha señalado que el programa de acompañamiento para 
pueblos indígenas de la UNSa no bastaba para equiparar las trayectorias universitarias de los estudiantes indígenas con la trayectoria teórica estipulada para los estudiantes universitarios en Argentina — cinco años de cursado ininterrumpido y egreso inmediatamente luego de cumplir con el cursado- (Ossola, 2015). Sin embargo, la tutoría cumple un rol importante para mantener, a lo largo de los años, el vínculo entre la institución, los estudiantes autorreconocidos como originarios y los tutores-pares.

Una egresada que se desempeñó como tutora, comenta el impacto de su paso por las tutorías en su posterior desempeño como docente:

[...] esta tutoría ha cambiado incluso mi camino de formación docente $y$, siendo tutora luego $[. .$.$] ya me recibí, y voy consiguiendo$ otros trabajos y todo esto, pero al fin y al cabo termino volviendo, por así decirlo, nunca me termino yendo de la Universidad por este mismo vínculo con los chicos, con el espacio, con el servicio... con la tutoría... (Colaboradora y extutora del ProTconPO)

Si bien la tutoría plantea el acompañamiento para fortalecer el rendimiento académico de las y los estudiantes, en este testimonio la joven señala de qué manera su experiencia como tutora influyó en posteriores decisiones profesionales, ya que el vínculo con los estudiantes indígenas profundizó sus deseos de desempeñarse como profesora. En este caso, la tutoría favoreció la retención y orientó la inserción laboral de una extutora, una función no contemplada en sus bases, pero importante en el actual contexto de incertidumbre respecto a las trayectorias universitarias y laborales de los estudiantes, más allá de sus identificaciones étnicas.

La egresada también señala que las tutorías conforman un lugar pedagógico, un "sitio de aprendizaje" al que siempre termina volviendo, dando cuenta de que el apoyo social y la contención no es unidireccional (tutores-estudiantes), sino que opera en múltiples direcciones (entre tutores, tutores y estudiantes, tutores, estudian- tes y coordinación, coordinación y estudiantes, etc.). Como espacio formativo, se vislumbra que las tutorías generan prácticas que luego serán motivadoras para insertarse en otros ámbitos de la universidad, como la docencia.

[...] ya muchos [estudiantes indígenas que han participado de las tutorías] ya son tutores, ya muchos son auxiliares adscriptos, y que vos ves que ese rol va acompañado de otras cosas también, que las tutorías, tal vez han proporcionado, este otro espacio, de ver por el otro... eh... vos decís 'bueno, creo que las expectativas o ciertos objetivos se cumplieron'. (Colaboradora y extutora del ProTconPO)

Ello muestra una arista que no forma parte de los objetivos iniciales del proyecto, que es el contacto con ciertas formas de enseñanza y aprendizaje, y con contenidos que resultan formativos para ejercer la docencia con un estilo que promueve la empatía.

\subsection{Tutoría y revalorización de lo comunitario}

La administración del tiempo para el estudio y para la realización de otras actividades - principalmente ligadas al ámbito comunitario- son temas de reflexión y debate los espacios de tutoría. Uno de los ejes para entender las formas de habitar la universidad por parte de los estudiantes indígenas es comprender la complementariedad entre las actividades que se realizan en la ciudad (entre ellas, el estudio en la universidad) y las que se desarrollan en la comunidad:

[...] cuando se van por ejemplo a sus casas, em... el hecho de juntar plata, de trabajar en las cosechas, de ir ... o las vacaciones, es el momento de volver a colaborar con el grupo familiar y con la comunidad. (Colaboradora y ex-tutora del ProTconPO)

Es necesario destacar el papel que tiene la comunidad al momento de seleccionar a los jóvenes que irán a la universidad y las carreras que van a cursar, en base a lo que comunitaria- 
mente se torna deseable o necesario (Czarny, Ossola, \& Paladino, 2018). Existen carreras que gozan de consenso entre las comunidades originarias: las ligadas al ámbito de la salud (medicina o enfermería), las relacionadas con la educación formal (maestro o profesor) y el derecho - que representa la posibilidad de defender a las comunidades frente a las amenazas de la sociedad mayoritaria-. De este modo, estudiar en la universidad guarda significados diversos, que incluyen aspectos como la posibilidad de experimentar nuevas oportunidades de vida en la ciudad, cumplir con las expectativas comunitarias, regresar a la comunidad para dar cuenta de los roles asumidos anteriormente y obtener un título universitario (Czarny et al., 2018).

Desde la perspectiva de los jóvenes indígenas, se destacan tensiones entre la disposición hacia el ejercicio de una carrera por gusto personal y las demandas de las comunidades. Al respecto, nos comenta una tutora par:

Como tutora me pasó a mí de que una estudiante realmente brillante de la Licenciatura en Matemática, que estaba regularizando todo y promocionando las materias que eran promocionales en su carrera. Ella se cambió de carrera a [la Licenciatura en] Antropología. Totalmente diferente. Y viene y me dice: 'me voy a cambiar', y yo '¿qué te vas a cambiar?', '[voy a cambiar] de carrera, no aguanto más' dice así. Y para mí fue un baldazo de agua fría, que venga una persona a decirte que va a cambiar el rumbo de su vida. Se cambió a antropología, y le digo 'bueno, si a vos te hace bien', me planteó porqué se cambiaba, eh... hubo muchas cosas en la comunidad que la hicieron cambiar de opinión. También los encuentros con el grupo acá, entonces empezó a preguntarse. La apasiona la matemática a ella, o sea eso, eso no lo puede negar, pero realmente ella no se ve como una profesional de la matemática, no ve cómo contribuir [desde la matemática], ella quiere contribuir a la comunidad, [...] sí, dice, como docente [de matemática] tal vez, si estoy en la comunidad o en un profesorado de allá, pero no, no le ve como un 'más allá. Entonces se cambió a antropología, y el primer año de antropología seguía yendo al servicio [tutoría] en [la Facultad de Ciencias] Exactas, creo que sigue yendo incluso, pasa por el servicio, y... está realmente fascinada con la carrera, los primeros viajes, porque desde el primer año hacen viajes [...] me dice que ha confirmado que era antropología lo que ella quería, que nunca se había dado cuenta. (Colaboradora y extutora del ProTconPO).

A través del relato de la tutora se accede a una reconstrucción de sentidos que una estudiante realizó sobre su paso por dos carreras diferentes en la misma universidad. Primero, se inscribió en la Licenciatura en Matemática, la carrera por la que tenía una preferencia explícita y en la que demostraba muy buen rendimiento. Conforme fue pasando el tiempo, comenzó a conocer nuevos campos de conocimiento en la universidad y en la comunidad, y visualizó la Licenciatura en Antropología, como un ámbito en el cual podía conjugar diferentes expectativas: la de recibir un título universitario, la de sentirse satisfecha con la elección y la de colaborar con su comunidad.

Se encuentra en esta trayectoria una forma propia de unir las demandas que diferentes sectores ejercen sobre las decisiones de los y las jóvenes indígenas. En este caso, el rol de la tutora universitaria fue importante para que la joven pueda expresar sus estados de ánimo frente a los cambios, y sentir que lo que eligiera iba a ser aceptado y valorado desde el espacio de las tutorías.

\subsection{Tutorías y CEUPO}

Los estudiantes que participan y conforman las tutorías, se constituyeron en un grupo autodenominado CEUPO (Comunidad de Estudiantes Universitarios de Pueblos Originarios) y desde allí realizan actividades conjuntas. El CEUPO se presenta como:

Un grupo de estudiantes universitarios que lucha por el reconocimiento y el respeto a la 
diversidad cultural en nuestra Universidad. En este grupo participamos en forma voluntaria estudiantes de las diferentes facultades, compartiendo saberes, conocimientos y experiencias de nuestras culturas y la vida en la Universidad. Somos una comunidad que tiene voz, somos eco de nuestras culturas ancestrales, con identidad, con historia; somos una comunidad que se abre caminos en esta universidad (Tomado de la página de Facebook del CEUPO).

El CEUPO es un espacio importante para mantener y sostener las identificaciones étnicas de los estudiantes indígenas durante la realización de sus carreras de grado:

La Universidad para mí es una casa enorme y bueno, el CEUPO sería la comunidad nuestra, sería [el lugar de] nuestras culturas, donde están como nuestras creencias, nuestros saberes, nuestras maneras, tal vez, de ver el mundo, pero dentro de otra institución. Así que para mí el CEUPO es como una familia, una familia dentro de la Universidad, una casa más, una pequeña casa que están todas las culturas, que nos permite conocernos, que nos permite mantener la cultura, nuestra identidad, nuestra manera de pensar. (Extutora del ProTconPO, proveniente de una comunidad indígena).

El CEUPO, nacido de las tutorías, constituye desde la perspectiva de la entrevistada, el espacio en el que se recrean los vínculos de cercanía y familiaridad: "la casa" dentro de la universidad. Esto da cuenta de la eficacia del programa en su búsqueda por lograr la contención socio-afectiva, allende el rendimiento académico.

\subsection{Experiencias formativas y diversi- dad lingüística}

El acceso a mayores niveles de escolaridad entre los pueblos indígenas suele asociarse a la pérdida de la lengua materna y su desplazamiento por el uso mayoritario de las lenguas estatales o hegemónicas (Hecht, 2010). No obstante, entre los jóvenes del pueblo wichí que cursan estudios de grado en la UNSa, se ha señalado que el alto grado de vitalidad lingüística en su lengua opera como un mecanismo de contención grupal en la etapa de ingreso en la universidad (Ossola, 2015). Asimismo, en relación con las tutorías, aparecen importantes desafíos en torno a los modos de construcción y transmisión de conocimientos en contextos plurilingües:

Los textos te hablan de conceptos que en su lengua materna no existen. Entonces, es tratar de encontrar y de contextualizar esa palabra. Eh... es un trabajo muy abstracto y es un triple o cuatro veces mayor el esfuerzo que tenés que hacer para comprender un texto, para leer un texto, leer una página de un texto. Entonces es un trabajo muy individual, agotador diría yo, cansador y hasta frustrante para el estudiante. Em... tenemos el caso de uno de los chicos wichí en [la Licenciatura en] enfermería, que tiene [la materia] Introducción a la Enfermería, que son unos textos bien largos sobre el cuidado enfermero, sobre ciertos conceptos del cuidado enfermero, em... entonces, es ir viendo de cómo... (Colaboradora y extutora del ProTconPO)

La cita refleja las singularidades que tienen la enseñanza y el aprendizaje en contextos de diversidad cultural y lingüística. En este tipo de situaciones son replanteadas las formas de transmitir conocimientos, - pues deben deconstruirse las ideas previas en torno a un concepto académico-, las maneras y los estilos del acompañamiento tutorial, y la mirada propia en torno de qué es aprender en la universidad.

\section{Discusión y conclusiones}

El estudio cualitativo realizado permite iniciar un acercamiento a los significados complejos que reviste el formar parte del espacio de tutorías para estudiantes indígenas en la Universidad Nacional de Salta. Tomando en cuenta la intensidad de las relaciones que allí se producen y las 
lógicas propias de organización y transmisión de los conocimientos (académicos y no académicos) que se dan en su interior, proponemos concebir a las tutorías universitarias para estudiantes indígenas como comunidades de práctica, es decir, como organizaciones sociales con pautas y objetivos propios, en las que sus participantes se organizan a través de diferentes actividades para el traspaso de habilidades y saberes específicos (Lave, \& Wenger, 1991).

En la cotidianeidad de las tutorías se pueden observar las múltiples aristas de la formación académica, aquello que los sujetos ponen en juego, sus expectativas y construcciones de futuro. En el caso particular del acompañamiento a estudiantes indígenas, las tutorías funcionan como un espacio intermedio entre la rigidez de las estructuras universitarias y la familiaridad de las prácticas comunitarias. Debido a ello, devienen un espacio apto para analizar las huellas que la educación ha dejado en los estudiantes, tanto en lo formal como en lo familiar-comunitario.

Las tutorías para pueblos indígenas reflejan las grandes tensiones de la construcción identitaria, profesional y comunitaria de los jóvenes que participan en ella. La interculturalidad es vivenciada desde lo cotidiano como un trabajo incesante por traducir significados y pertenecer a diferentes espacios: la comunidad, la familia, las tutorías, las carreras de grado, etc.

Uno de los grandes desafíos que se presentan en este ámbito es la interacción en el marco de la diversidad lingüística. En este sentido, los estudiantes que provienen de contextos con pronunciada vitalidad de la lengua indígena enfrentan un doble extrañamiento: frente al modo académico de aprendizaje y frente al español como lengua para adquirir los conocimientos. Ante estos desafíos, los tutores cumplen un rol principal al dar cuenta de las situaciones multilingües y al acompañar personalmente a los estudiantes bilingües.

También se destaca que la inclusión no es unidireccional: se traza constantemente en movimientos de vaivén, partiendo de situaciones reales en las cuales los estudiantes y los tutores confrontan sus saberes y se acercan y alejan de la institución universitaria según las particulares condiciones individuales, familiares y comunitarias que atraviesan.

El estudio de caso presentado permite dar cuenta de un ámbito específico, pero los aprendizajes que de allí se desprenden resultan significativos para pensar la compleja realidad que vivencian las y los estudiantes universitarios latinoamericanos en el contexto actual.

\section{Apoyos y agradecimientos}

Esta investigación es posible gracias al financiamiento del Consejo Nacional de Investigaciones Científicas y Técnicas (CONICET). Cuenta con el apoyo del Proyecto Institucional "Territorialidad y poder. Conflictos, exclusión y resistencias en la construcción de la sociedad en Salta", del Instituto de Investigaciones en Ciencias Sociales y Humanidades (ICSOH), y del proyecto de investigación "Impacto de las transformaciones recientes en las comunidades indígenas del Chaco Salteño: reconfiguraciones identitarias, nuevas modernidades y políticas públicas" (Consejo de Investigación de la Universidad Nacional de Salta).

Se agradece especialmente a los miembros del proyecto de tutorías con los estudiantes de pueblos originarios de la UNSa, quienes han compartido generosamente sus experiencias.

\section{Notas}

1 Cruce de datos del Censo 2010 con los de ENOTPO (Encuentro Nacional de Organizaciones Territoriales de Pueblos Originarios, 2015), realizado por Elisa Sulca y Nuria Rodríguez (2019) en el marco de sus tesis doctorales.

2 Este estudio de caso se realiza en el marco de la Tesis Doctoral en curso de Nuria M. Rodríguez, titulada "Interculturalidad y Universidad. Desarrollo, avances y perspectivas de los proyectos de tutoría con estudiantes de pueblos originarios en la Universidad Nacional de Salta". Doctorado en Educación. Universidad Nacional de Tucumán. 


\section{Referencias bibliográficas}

Aikman, S. (1999). La educación indígena en Sudamérica. Interculturalidad y bilingüismo en Madre de Dios, Perú. Lima, Perú: IEP ediciones.

Briones, C. (2005). Formaciones de alteridad: contextos globales, procesos nacionales y provinciales. En C. Briones (Comp.), Cartografías argentinas. Politicas indigenistas y formaciones provinciales de alteridad. Buenos Aires: Antropofagia.

Briones, C., Delrio, W., Lanusse, P. Lazzari, A. Lorenzetti, M. Szulk, A., \& Vilvaldi, A. (2006). Diversidad cultural e interculturalidad como construcciones socio-históricas. En A. Amegeiras y E. Jure (Comps.), Diversidad cultural e interculturalidad (pp. 255-264). Buenos Aires: Prometeo libros y Universidad Nacional de General Sarmiento.

Capelari, M. I. (2016). El rol del tutor en la Universidad: configuraciones, significados y prácticas. Buenos Aires: SB.

Carli, S. (2012). El estudiante universitario. Hacia una historia del presente de la educación pública. Buenos Aires: Siglo XXI Editores.

Claro, M., \& Seoane, V. (2005). Acción afirmativa. Hacia democracias inclusivas. Santiago de Chile: Fundación Equitas.

Coronado, M., \& Gómez Boulin, M. J. (2015). Orientación, tutorías y acompañamiento. En Educación Superior. Análisis de las trayectorias estudiantiles. Los jóvenes ante sus encrucijadas. Buenos Aires: Noveduc.

Czarny, G. (2008). Pasar por la escuela. Indígenas $y$ procesos de escolaridad en la ciudad de México. Distrito Federal, México: Universidad Pedagógica Nacional.

Czarny, G., Ossola, M. M., \& Paladino, M. (2018). Jóvenes indígenas y universidades en América Latina: sentidos de la escolaridad, diversidad de experiencias y retos de la profesionalización. Antropología Andina, 5(1).

Delgado, M. (2002). El poder de la clasificación. El inmigrante como discapacitado cultural. En J. García Molina Y R. M. Ytarte (Eds.), Pedagogía social y mediación educativa. La Mancha, Toledo: Universidad de Castilla.
Delory-Momberger, C. (2009). Biografía y educación: figuras del individuo-proyecto. Buenos Aires: Editorial de la Facultad de Filosofía y Letras UBA.

Diez, C., García, J., Montesinos, M. P., Pallma, S., \& Paoletta, H. (2015). Discutiendo categorías... acerca de los usos (y abusos) de los términos inclusión y exclusión. Boletín de Antropología y Educación (9), 33-39. (goo.gl/4pCX4U) (2019-01-28).

Diez, M. L. (2004). Reflexiones en torno a la interculturalidad. Cuadernos de Antropología social (19), 191-213. (goo.gl/PBhvV) (2019-01-28).

Grupo de Investigación: Hum 0109 (1997). "Introducción al Estudio de Caso". Documento de Trabajo Interno $\mathrm{N}^{\circ} 4$. Andalucía.

Guaymás, A. (2016). Educación superior y pueblos indígenas: políticas y prácticas de reconocimiento de la diversidad cultural e interculturalidad en Argentina. En María Verónica Di Caudo, Daniel Llanos Erazo y María Camila Ospina (Coords.), Interculturalidad y educación desde el Sur. Contextos, experiencias y voces. Quito: Editorial Universitaria Abya-Yala.

Hanne, A. (2018). Estudiantes indígenas y universidad: realidades y retos ante la diversidad cultural. Caso de la Universidad Nacional de Salta. Alteridad, 13(1), 14-29. https://doi.org/10.17163/alt.v13n1.2018.01.

Hecht, A. (2010). 'Todavía no se hallaron en hablar en idioma'. Procesos de socialización lingüística de los niños en el barrio toba de Derqui. Múnich: Lincom Europa.

Ilvento, M.C., Martínez, M.T., Rodríguez, J., \& Fernández Berdaguer, L. (2011). Trayectorias educativas e inserción laboral: un encuentro de miradas. Salta: Mundo Gráfico.

Instituto Nacional de Estadísticas y Censos (INDEC) (2001).Censo Nacional de Población, Hogares y Viviendas 2001. República Argentina.

Instituto Nacional de Estadísticas y Censos (INDEC) (2010).Censo Nacional de Población, Hogares y Viviendas 2010. República Argentina.

Lave, J., \& Wenger, E. (1991). Situated Learning. Legitimate peripheral participation. Nueva York: Cambridge University Press.

Lobato Fraile, C. \& Ilvento, M. C. (2013). La Orientación y tutoría universita- 
ria: una aproximación actual. Revista de Docencia Universitaria, 11(2), 17-25. https://doi.org/10.4995/redu.2013.5564

Luciano, G. (2009). Indígenas no Ensino Superior: Novo Desafio para as Organizações Indígenas e Indigenistas no Brasil. En $53^{\circ}$ Congreso Internacional de Americanistas. México, D. F.

Luján, A., Soto, M., \& Rosso, L. (2018). Experiencias de ingreso y permanencia de estudiantes indígenas en una universidad convencional del nordeste argentino. Antropología Andina, 5(1), pp. 43-66.

Mikkelsen, C. (Comp/Ed.) (2014). El mundo indígena 2014. Copenhague: IWGIA (Grupo Internacional de Trabajo sobre Asuntos Indígenas).

Möller, A. (2012). What is Compensatory Pedagogy Trying to Compensate for?. Issues in Educational Research, 22 (1), 60-78. (goo.gl/FxTmWP) (2019-01-28).

Ossola, M. M. (2015). Aprender de las dos ciencias. Etnografía con jóvenes wichí en la educación superior de Salta. Santiago del Estero: EDUNSE.

Ossola, M. M. (2016). Pueblos indígenas y educación superior en la Argentina: debates emergentes. Revista del Cisen Tramas/Maepova, 4(1), 57-77. (goo.gl/Z4H3Dy) (2019-01-28).

Paladino, M. (2006). Estudar e experimentar na cidade: Trajetórias sociais, escolarização $e$ experiência urbana entre "Jovens" indígenas ticuna, Amazonas. Tesis de doctorado Antropología. Programa de Posgraduación de la Universidad Federal de Río de Janeiro. Río de Janeiro [obra inédita].

Paladino, M. (2009). Pueblos indígenas y educación superior en Argentina. Datos para el debate. Revista ISEES, (06), 81-122. Santiago, Chile:
Fundación Equitas. (goo.gl/7zHzMY) (201901-28).

Reygadas, L. (2007). La desigualdad después del (multi)culturalismo. En A. Giglia, C. Garma y A. P. De Teresa (Comps.), ¿A dónde va la antropología? México: Universidad Autónoma Metropolitana-Iztapalapa.

Rezaval, J. (2008). Políticas de inclusión social a la educación superior en Argentina, Chile y Perú. Buenos Aires: FLACSO.

Sánchez Martínez, E. (2003). La legislación sobre Educación Superior en Argentina. Entre rupturas, continuidades y transformaciones. Documento de trabajo (102). Buenos Aires: Universidad de Belgrano.

Stake, R. E. (1995). The Art of Case Study. London: Sage.

Terigi, F. (2008). En la perspectiva de las trayectorias escolares. Comentario al capítulo 3 del atlas de las desigualdades educativas en América Latina: "La asistencia escolar en la actualidad. Trayectorias educativas en 8 países de América Latina". Buenos Aires: SITEALIIPE- UNESCO Sede Regional Buenos Aires.

UNICEF (Fondo de la Naciones Unidas para la Infancia) (2009). Los pueblos indígenas en Argentina y el derecho a la educación. Situación socioeducativa de niñas, niños $y$ adolescentes de comunidades rurales wichí y mbyá guaraní. Buenos Aires: UNICEF.

Wigdorovitz de Camilloni, A. R. (2008). El concepto de inclusión educativa: definición y redefiniciones. Politicas Educativas-Campinas, 2(1), 1-12. (goo.gl/bUJrWw) (2019-01-28).

Yuni, J., \& Urbano C. (2016). Técnicas para investigar: recursos metodológicos para la preparación de proyectos de investigación. Córdoba: Brujas. 